\title{
Interleukin-17A facilitates osteoclast differentiation and bone resorption via activation of autophagy in mouse bone marrow macrophages
}

\author{
LU SONG* , JINGYITAN*, ZHONGXIU WANG, PEIHUI DING, QI TANG, \\ MENGJIAO XIA, YINGMING WEI and LILI CHEN \\ Department of Periodontology, The Second Affiliated Hospital of Zhejiang University School of Medicine, \\ Hangzhou, Zhejiang 310009, P.R. China
}

Received September 29, 2018; Accepted April 5, 2019

DOI: $10.3892 / \mathrm{mmr} .2019 .10155$

\begin{abstract}
Interleukin 17A (IL-17A) exerts pleiotropic effects on periodontitis, partially through enhancement of alveolar bone loss. Osteoclasts are the main culprits that absorb alveolar bone. However, studies describing the correlation between IL-17A and osteoclasts are not conclusive. Previously, autophagy was revealed to be involved in osteoclast differentiation and bone resorption. However, the role of autophagy in IL-17A-mediated osteoclast formation is yet to be clarified. In the present study, bone marrow macrophages (BMMs) were treated with or without IL-17A. 3-Methyladenine (3-MA) was applied to inhibit autophagy. Osteoclast formation was detected by tartrate-resistant acid phosphatase (TRAP) staining, immunofluorescence, and scanning electron microscope. The effects of IL-17A on osteoclast-specific genes and autophagy-related genes during osteoclast differentiation were examined by real-time quantitative polymerase chain reaction and western blot analysis. Autophagosomes were observed by transmission electron microscope. Hematoxylin and eosin (H\&E), and TRAP staining was adopted to assess alveolar bone destruction and the number of osteoclasts, respectively
\end{abstract}

Correspondence to: Professor Lili Chen, Department of Periodontology, The Second Affiliated Hospital of Zhejiang University School of Medicine, 88th Jiefang Road, Hangzhou, Zhejiang 310009, P.R. China

E-mail: chenlili_1030@zju.edu.cn

*Contributed equally

Abbreviations: IL-17A, interleukin 17A; BMMs, bone marrow macrophages; 3-MA, 3-methyladenine; TRAP, tartrate-resistant acid phosphatase; NFATc1, nuclear factor of activated $\mathrm{T}$ cells 1; CatK, cathepsin K; RANKL, receptor activator of nuclear factor- $\kappa \mathrm{B}$ ligand; LC3, microtubule-associated protein 1 light chain 3; M-CSF, macrophage colony-stimulating factor

Key words: interleukin 17, osteoclasts, bone resorption, autophagy, periodontitis in a rat periodontitis model. Consequently, IL-17A stimulated osteoclast differentiation and bone resorption of BMMs accompanied by an increase in the mRNA expression of osteoclast-specific genes. Furthermore, IL-17A increased the levels of autophagy-related genes and proteins, and inhibition of autophagy with 3-MA attenuated the IL-17A-mediated osteoclastogenesis. In addition, there was an increase in the number of osteoclasts and alveolar bone resorption with IL-17A treatment in the periodontitis rat model. Collectively, these findings indicated that IL-17A facilitated osteoclast differentiation and bone resorption in vitro and in vivo, which may contribute to the understanding of the molecular basis of IL-17A in alveolar bone destruction and provide insight on the clinical therapeutic targets for periodontitis.

\section{Introduction}

Periodontitis is a chronic inflammatory disease with periodontal ligament inflammation and alveolar bone destruction (1). It is initiated by bacterial plaque, which induces inflammatory immune responses to perturb the bone homeostasis between bone construction and bone resorption (2). Although several studies have explored the complex regulatory mechanisms underlying alveolar bone loss, in the pathogenies of periodontitis, these are unclear (3).

Interleukin-17A (IL-17A) is a pro-inflammatory cytokine typically secreted by Th17 cells and contributes to the pathophysiology of periodontitis, notably aggravating gingival inflammation and alveolar bone loss (4). Emerging clinical studies have revealed that human periodontitis is related to the increased expression of IL-17 in peripheral blood, gingival crevicular fluid, gingival tissues, and alveolar bone when compared to a healthy periodontal group (5-7). Additionally, the increased number of IL-17-positive cells and osteoclasts were accumulated in the alveolar bone of periodontitis patients, indicating a causal relevance of IL-17 and osteoclasts in periodontitis (8). However, the underlying mechanism of IL-17A-mediated inflammatory periodontal bone destruction is yet to be elucidated.

Furthermore, osteoclasts are specialized multinucleated cells derived from macrophage precursors and activated to 
absorb the alveolar bone, ultimately resulting in bone loss in periodontitis (9). Notably, IL-17A was revealed to possess valid osteoclastogenic properties that stimulate the expression of the receptor activator of nuclear factor $-\kappa B$ ligand (RANKL), interleukin $1 \beta$ (IL-1 $\beta$ ), tumor necrosis factor- $\alpha$ (TNF- $\alpha$ ), and prostaglandin E2. Of note, the targets of IL-17A contain osteoblasts, fibroblasts, and other stromal cells, indicating that IL-17A mediates osteoclast activity by promoting osteoclastogenesis factors on other cells (10-12). However, the study of osteoclast differentiation under the direct effect of IL-17A on osteoclast precursors is poorly understood.

Autophagy is an intracellular metabolic pathway, which orchestrates diverse aspects of cellular stress response, and the core molecular machinery of autophagy consists of autophagy-related proteins $(13,14)$. Recently, autophagy has been demonstrated to be involved in osteoclast differentiation and bone resorption $(15,16)$. Autophagy-related proteins, including Atg5, Atg7, Atg4B, and microtubule-associated protein 1 light chain 3 (LC3) are significantly responsible for the formation of ruffled border and facilitation of osteoclast polarization, ultimately resulting in bone resorption (17). Moreover, emerging evidence has indicated that several pro-inflammatory cytokines, including IL- $1 \beta$ and TNF- $\alpha$, trigger autophagy in murine osteoclasts $(18,19)$. However, whether autophagy is involved in IL-17A-mediated osteoclastogenesis requires further exploration.

In the present study, the effects of IL-17A on osteoclast differentiation and bone resorption of BMMs were investigated, and whether autophagy was involved in this process was analyzed. Moreover, the effect of IL-17A on osteoclast activity was detected on the alveolar bone surfaces in a rat periodontitis model.

\section{Materials and methods}

Materials. Soluble recombinant mouse IL-17A was obtained from PeproTech, Inc. (Rocky Hill, NJ, USA). Macrophage colony-stimulating factor (M-CSF) and RANKL were obtained from R\&D Systems, Inc. (Minneapolis, MN, USA). All cell culture media and supplements were procured from Gibco; Thermo Fisher Scientific, Inc. (Waltham, MA, USA). Reagents for real-time quantitative polymerase chain reaction (PCR) were obtained from Takara Bio, Inc. (Otsu, Japan). RNA was extracted using TRIzol reagent from Thermo Fisher Scientific, Inc., according to the manufacturer's protocol. Antibodies against LC3B (cat. no. 192890; 1:2,000) and $\beta$-actin (cat. no. 8226; 1:1,000) were purchased from Abcam (Cambridge Science Park, Cambridge, UK). An antibody against IL-17A (cat. no. GB11110; 1:100) was purchased from Servicebio (Wuhan, Hubei, China). Goat anti-rabbit immunoglobulin G secondary antibody (cat. no. BA1054; 1:5,000) was obtained from Boster Biological Technology, Ltd. (Wuhan, Hubei, China). A bicinchoninic acid (BCA) kit and enhanced chemiluminescence (ECL) detection reagent was purchased from Thermo Fisher Scientific, Inc. TRAP staining was conducted using a Leukocyte Acid Phosphatase kit (Sigma-Aldrich; Merck KGaA, Darmstadt, Germany). Cell viability was assessed using Cell Counting Kit-8 (CCK-8) (Sigma-Aldrich; Merck KGaA).
Animals. All animal experiments were performed in accordance with the principles and procedures of the National Institutes of Health (NIH) for the Care and Use of Laboratory Animals. The approval number granted by the Animal Ethics Committee of The Second Affiliated Hospital of Zhejiang University School of Medicine is 2017-052 (2017-2-10). All surgeries were performed under sodium pentobarbital anesthesia, and all efforts were made to minimize suffering. A total of 10 female 7-week-old C57BL/6 mice $(22 \pm 2 \mathrm{~g})$, and 30 male 7-weeks of age Sprague-Dawley rats $(200 \pm 50 \mathrm{~g})$, were purchased from the China Experimental Animal Center (Hangzhou, China). Throughout the experiment, the animals were housed in individual cages under an appropriate temperature $\left(21 \pm 2^{\circ} \mathrm{C}\right)$, with 12 -h light/dark cycles and humidity $(50 \pm 5 \%)$ and fed standard laboratory food and tap water ad libitum.

Cell culture. BMMs were obtained as previously described (20). Briefly, C57BL/6 female mice were euthanized by cervical dislocation after being anesthetized with sodium pentobarbital (150 mg/kg body weight, intraperitoneally). The femora and tibiae were dissected from mice, and the ends were cut off with scissors. Then, a 1- $\mu 1$ syringe was used to flush the marrow cavity with $\alpha$-MEM medium supplemented with $10 \%$ fetal bovine serum, $100 \mathrm{U} / \mu 1$ penicillin, $100 \mathrm{U} / \mu 1$ streptomycin, and $30 \mathrm{ng} / \mu \mathrm{l} \mathrm{M}-\mathrm{CSF}$. The mixture was homogenized and incubated in a Petri dish at $37^{\circ} \mathrm{C}$ and $5 \% \mathrm{CO}_{2}$ for 3 days. The adherent cells were collected as BMMs.

Osteoclast formation. BMMs were seeded into 96-well plates at a density of $1 \times 10^{4}$ cells/well in the presence of M-CSF (30 ng/ $/ 1$ ), RANKL (50 ng/ $\mu \mathrm{l})$, and different concentrations of recombinant IL-17A $(0,0.1,1$ or $10 \mathrm{ng} / \mu \mathrm{l})$. The media were changed every alternate day until multinucleated cells appeared under a Leica light microscope (Leica, Solms, Germany).

TRAP staining. When the multinucleated osteoclasts were formed, the medium was aspirated, the cells were washed with phosphate-buffered saline (PBS), fixed with $4 \%$ paraformaldehyde at room temperature for $20 \mathrm{~min}$, and stained for TRAP using a leukocyte acid phosphatase kit. After the staining, multinucleated osteoclasts which contained 3 or more nuclei, could be identified by counting the number of nuclei in an osteoclast using a Leica light microscope (Leica).

Bone resorption assay in vitro. BMMs were seeded on bone slices at a density of $1 \times 10^{4}$ cells/slice in 96-well culture plates in 3 replicates in the presence of $30 \mathrm{ng} / \mu \mathrm{l} \mathrm{M-CSF}$. After $24 \mathrm{~h}$, the cells were treated with $30 \mathrm{ng} / \mu 1 \mathrm{M}-\mathrm{CSF}, 50 \mathrm{ng} / \mu 1 \mathrm{RANKL}$, and different concentrations of IL-17A $(0,0.1,1$ and $10 \mathrm{ng} / \mu \mathrm{l})$. The medium was replaced every alternate day until the formation of mature osteoclasts. Then, the bone slices were removed, washed with PBS, and dried. The bone resorption pits were observed using a scanning electron microscope (TM-100; Hitachi, Ltd., Tokyo, Japan).

Detection of F-actin ring formation. After mature osteoclasts formed, the cells were fixed with $4 \%$ paraformaldehyde at room temperature for $20 \mathrm{~min}$, permeabilized with $0.1 \%$ Triton $\mathrm{X}-100$ at room temperature for $10 \mathrm{~min}$, blocked with $1 \% \mathrm{BSA}$ 
for $30 \mathrm{~min}$, and incubated with TRITC-labeled phalloidin (Invitrogen; Thermo Fisher Scientific, Inc.,) for $2 \mathrm{~h}$. DAPI was used for staining the cell nuclei. Subsequently, the F-actin ring formation was observed using a Leica fluorescence microscope (Leica).

Transmission electron microscopy. Cells were fixed with $2.5 \%$ glutaraldehyde for $30 \mathrm{~min}$, and post-fixed in $1 \%$ buffered osmium tetroxide for $1 \mathrm{~h}$ at $20^{\circ} \mathrm{C}$. Then, the samples were dehydrated by an increasing ethanol gradient and embedded in epoxy resin. Ultrathin sections (60-80 $\mathrm{nm}$ ) were sliced using an ultramicrotome (Leica EM UC7; Leica), and observed under a transmission electron microscope (Tecnai G2 20 TWIN; FEI; Thermo Fisher Scientific, Inc.).

Cell viability assay. BMMs were plated in 96-well plates ( $1 \times 10^{3}$ cells/well) for $24 \mathrm{~h}$. Then, the cells treated with IL-17 were incubated with or without 3-MA (2 nM) for $48 \mathrm{~h}$. Cell viability was assessed using a CCK- 8 assay. Briefly, $10 \mu \mathrm{l}$ of CCK- 8 solution was added to each well at the indicated time-points, and then the plate was incubated for $2 \mathrm{~h}$ in a $5 \% \mathrm{CO}_{2}$ incubator. Absorbance was assessed at $450 \mathrm{~nm}$ with a spectrophotometer (Bio-Rad Laboratories, Inc., Hercules, CA, USA).

RNA extraction and real-time quantitative polymerase chain reaction. Total RNA of the cells was extracted using TRIzol reagent (Invitrogen; Thermo Fisher Scientific, Inc.) and reverse transcribed to synthesize single-stranded cDNA using PrimeScript Reverse Transcription Master Mix Kit (Takara Bio, Inc.). The expression of the target genes was quantified using a SYBR polymerase chain reaction Master Mix Kit (Takara Bio, Inc.) on a StepOne Plus real-time polymerase chain reaction system (Applied Biosystems; Thermo Fisher Scientific, Inc.) according to the manufacturer's protocol. The relative expression level of the target genes was calculated using the relative quantitative method $2^{-\Delta \Delta \mathrm{Cq}}(21)$, and normalized to the mouse glyceraldehyde-3-phosphate dehydrogenase $(G A P D H)$ gene. The primer sequences are listed in Table I.

Western blot analysis. The total protein of the cells was extracted using cell lysate buffer and quantified using a bicinchoninic acid kit (Thermo Fisher Scientific, Inc.). An equivalent of $25 \mu \mathrm{g}$ of the total protein was resolved on a $12 \%$ gel using sodium dodecyl sulfate-polyacrylamide gel electrophoresis and transferred to a polyvinylidene fluoride (PVDF) membrane (Bio-Rad Laboratories, Inc.). Subsequently, the membrane was blocked with $5 \%$ skim milk at room temperature for $2 \mathrm{~h}$ and probed with the primary antibodies anti- LC3B (cat. no. 192890; 1:2,000) and anti- $\beta$-actin (cat. no. 8226; 1:1,000) purchased from Abcam. Incubation with the membranes was performed overnight at $4^{\circ} \mathrm{C}$. Then, the membrane was washed and incubated with a horseradish peroxidase-conjugated secondary antibody (1:5,000; cat. no. BA1054; Boster Biological Technology, Ltd.) at room temperature for $1.5 \mathrm{~h}$. The immunoreactive bands were visualized using an ECL kit, and the data were analyzed using ImageJ software, version 1.48 [National Institutes of Health (NIH), Bethesda, MD, USA].
Table I. Primers used for reverse transcription-quantitative polymerase chain reaction.

\begin{tabular}{ll} 
Gene symbol & \multicolumn{1}{c}{ Primer sequence (5'-3') } \\
\hline LC3 & F: CGGAGCTTTGAACAAAGAGTG \\
& R: TCTCTCACTCTCGTACACTTC \\
Beclin-1 & F: AGCTCAGTACCAGCGGGAGT \\
& R: TGGAAGGTGGCATTGAAGAC \\
Atg5 & F: TGTGCTTCGAGATGTGTGGTT \\
& R: GTCAAATAGCTGACTCTTGGCAA \\
c-Fos & F: CGGGTTTCAACGCCGACTA \\
& R: TTGGCACTAGAGACGGACAGA \\
NFATc1 & F: GGAGAGTCCGAGAATCGAGAT \\
& R: TTGCAGCTAGGAAGTACGTCT \\
CatK & F: CTCGGCGTTTAATTGGGAGA \\
& R: TCGAGAGGGAGGTATTCTGAGT \\
TRAP & F: CACTCCCACCCTGAGATTTGT \\
& R: CCCCAGAGACATGATGAAGTCA \\
GAPDH & F: GACTTCAACAGCAACTCCCAC \\
& R: TCCACCACCCTGTTGCTGTA
\end{tabular}

F, forward; R, reverse; LC3, microtubule-associated protein 1 light chain3; NFATc1, nuclear factor of activated T cells 1; CatK, cathepsin $\mathrm{K}$; TRAP, tartrate-resistant acid phosphatase; GAPDH, glyceraldehyde-3-phosphate dehydrogenase.

Experimental periodontitis induction. Sprague-Dawley rats were randomly divided into 3 experimental groups: NC (normal control), NS (normal saline) and the IL-17 group. To induce a rat periodontitis model, as previously described $(22,23)$, the rats were anesthetized with sodium pentobarbital $(40 \mathrm{mg} / \mathrm{kg}$ body weight) via intraperitoneal injection; 3.0 cotton threads were placed bilaterally around the intraorbital portion of the rat maxillary first molar to induce experimental periodontitis in NS and IL-17 groups, while the NC group was subjected to sham treatment. In addition, $20 \mu \mathrm{l}$ normal saline and $20 \mu \mathrm{l}$ recombinant IL-17A $(5 \mu \mathrm{g} / \mu \mathrm{l})$ were injected into the bilateral maxillary first molars of the rats every other day in the NS and IL-17 groups under anesthesia with sodium pentobarbital (40 $\mathrm{mg} / \mathrm{kg}$ body weight) via intraperitoneal injection, respectively. During the experiments, rats had no weight loss, no loss of appetite, no serious infection, and no weakness or sudden death.

Histomorphometric analysis and immunohistochemical staining. All the rats were euthanized after being anesthetized with sodium pentobarbital $(150 \mathrm{mg} / \mathrm{kg}$, intraperitoneally) at 1-month after injury induction and death was ascertained mainly based on complete cessation of a heartbeat. The maxilla was dissected carefully, immediately fixed in $4 \%$ paraformaldehyde for 1 day, and decalcified in $10 \%$ ethylenediaminetetraacetic acid buffer at room temperature for 6 weeks. The samples were embedded in paraffin, and $4-\mu \mathrm{m}$ thick sections were obtained in the proximal mandible for hematoxylin and eosin (H\&E) staining. The distance between the cementum-enamel junction (CEJ) and the alveolar callus 
(ABC) in the interproximal region of the maxillary first molar was observed using a Leica optical microscope (Leica). TRAP staining was performed using a leukocyte acid phosphatase kit to quantify the osteoclasts as described by the manufacturer. Multinucleated $\mathrm{TRAP}^{+}$cells on the alveolar bone surface around the first molar were enumerated as active osteoclasts. Immunohistochemical staining was performed to detect the level of IL-17A and LC3, using the respective antibodies (1:100 dilution) according to the manufacturer's protocol. Finally, the sections were observed under a Leica light microscope and positive cells were counted using ImageJ software (NIH).

Statistical analysis. All quantitative data are presented as the mean \pm standard deviation. Each experiment was performed in triplicate. Statistical analyses were performed using one-way analysis of variance (ANOVA), followed by Tukey's post hoc test with GraphPrism 7 software (GraphPad Software, Inc., La Jolla, CA, USA). P<0.05 was considered to indicate a statistically significant difference.

\section{Results}

IL-17A facilitates osteoclast differentiation and bone resorption of mouse bone marrow macrophages. To detect the effects of IL-17A on osteoclast differentiation and bone resorption, BMMs were cultured in medium with or without IL-17A. The hallmark of osteoclast differentiation is the formation of $\mathrm{TRAP}^{+}$multinucleated cells (24). The present results revealed that the number of $\mathrm{TRAP}^{+}$multinucleated cells was significantly increased with increasing concentration of IL-17A (Fig. 1A and D). In addition, the formation of filamentous actin (F-actin) rings is a characteristic of terminally differentiated osteoclasts, and bone resorption pits can be quantified for determining the bone loss (24). Immunofluorescence staining revealed that the circumference of the actin ring was larger as compared to the control groups, which indicated that increasing the areas of contact with the bone surface enhanced the function of bone resorption after the addition of IL-17A (Fig. 1C). In addition, the areas of the bone absorption pits were markedly promoted by IL-17A treatment (Fig. 1B and E). These results indicated increased osteoclastogenesis of BMMs in vitro after addition of IL-17A.

Effect of IL-17A on the expression of osteoclast-related genes in vitro. To further investigate the effects of IL-17A on osteoclast differentiation, the expression of osteoclast-related genes was examined by real-time quantitative PCR. c-Fos and NFATc1 are the osteoclastogenic transcription factors required for early osteoclast differentiation, while TRAP and CatK are designated as terminal osteoclast differentiation markers (24-26). Furthermore, slightly increased expression of c-Fos and NFATc1 was observed post-IL-17A treatment (Fig. 2A and B). Moreover, IL-17A significantly increased the expression TRAP and CatK as compared to the control group (Fig. 2C and D).

IL-17A facilitates autophagy activity during osteoclast differentiation. The specific mechanism underlying IL-17A-facilitated osteoclast differentiation was hypothesized to be the autophagic pathway. Thus, real-time quantitative
PCR was employed and it was revealed that the expression of the autophagy-related genes, LC3, Beclin1 and Atg5 was increased (Fig. 3A). Next, the expression of LC3-II protein, which is the autophagy marker in autophagosome membranes was investigated (14). Western blot analysis revealed that the expression of LC3-II was upregulated after the addition of IL-17A (Fig. 3B and C), indicating enhanced autophagosome formation. Notably, an increased number of autophagosomes with typical bilayer membrane morphology was observed by transmission electron microscopy (Fig. 3D and E). The aforementioned findings indicated that autophagy activity was enhanced in IL-17A-mediated osteoclast differentiation.

Inhibition of autophagy attenuates IL-17A-mediated osteoclastogenesis. To elucidate whether autophagy in IL-17A-treated BMMs exerted a positive effect, autophagy inhibitor 3-MA, a prominent inhibitor reducing autophagy flux in the early stages, was added. Consequently, no detrimental effect was noted on the viability of osteoclasts after the treatment with 3-MA (Fig. 4A). The data revealed that the expression of LC3-II was significantly downregulated with the treatment of 3-MA (Fig. 4B and E). Consecutively, the number of $\mathrm{TRAP}^{+}$multinuclear cells (Fig. 4C and F), the area of pits on bone slices (Fig. 4D and G), and the mRNA level of osteoclast-related genes, $C a t K$ and TRAP (Fig. 4H and I), were decreased after the addition of 3-MA in IL-17A-treated BMMs. These results demonstrated that the autophagy inhibitor could weaken the effect on IL-17A-mediated osteoclast activity.

Effect of IL-17A on osteoclast activity in vivo. A rat model of experimental periodontitis was established to detect the effect of IL-17A on osteoclasts. Immunohistochemistry demonstrated that a marked level of IL-17A in the periodontal tissue was detected after IL-17A injection when compared to normal saline injection (Fig. 5A and B). H\&E staining revealed that the attachments of the collagenous fibers were destroyed, and a large number of inflammatory cells were revealed in both the connective tissue and the epithelium layer in the NS and IL-17 groups when compared to the NC group. Additionally, a prominently enhanced average distance was noted between the CEJ and $\mathrm{ABC}$ in the IL-17 groups when compared to the NS group (Fig. 5C and D). In addition, TRAP staining exposed higher numbers of $\mathrm{TRAP}^{+}$multinuclear cells in the IL-17 groups (Fig. 5E and F). Moreover, an increase in LC3 staining was observed on the inflammatory alveolar bone surfaces after IL-17 treatment (Fig. 5G and H). Collectively, these results revealed that IL-17A could increase osteoclastogenesis to exacerbate alveolar bone resorption in the periodontitis rat model.

\section{Discussion}

In periodontitis, microbes and toxic products in bacterial plaques induce local inflammatory reactions in the periodontal tissues due to a large-scale production of inflammatory mediators, which can promote osteoclast activity and aggravate alveolar bone resorption (27). Accumulating evidence has revealed an interdisciplinary approach combing the study of bone and immune system, regarded as osteoimmunology, for the treatment of the disease (28). 
A

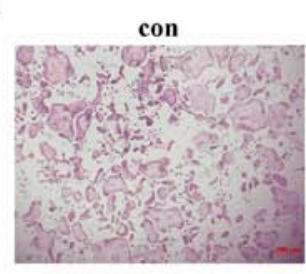

B
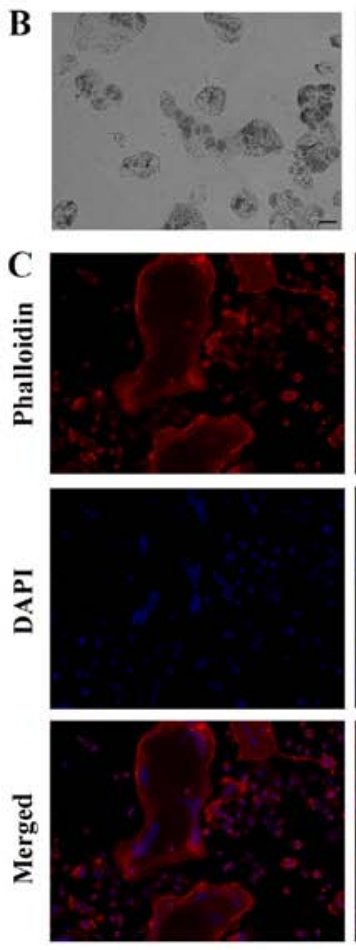

D

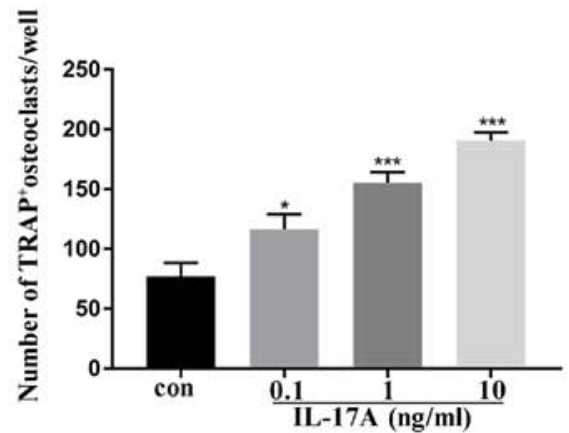

IL-17A (ng/ml)
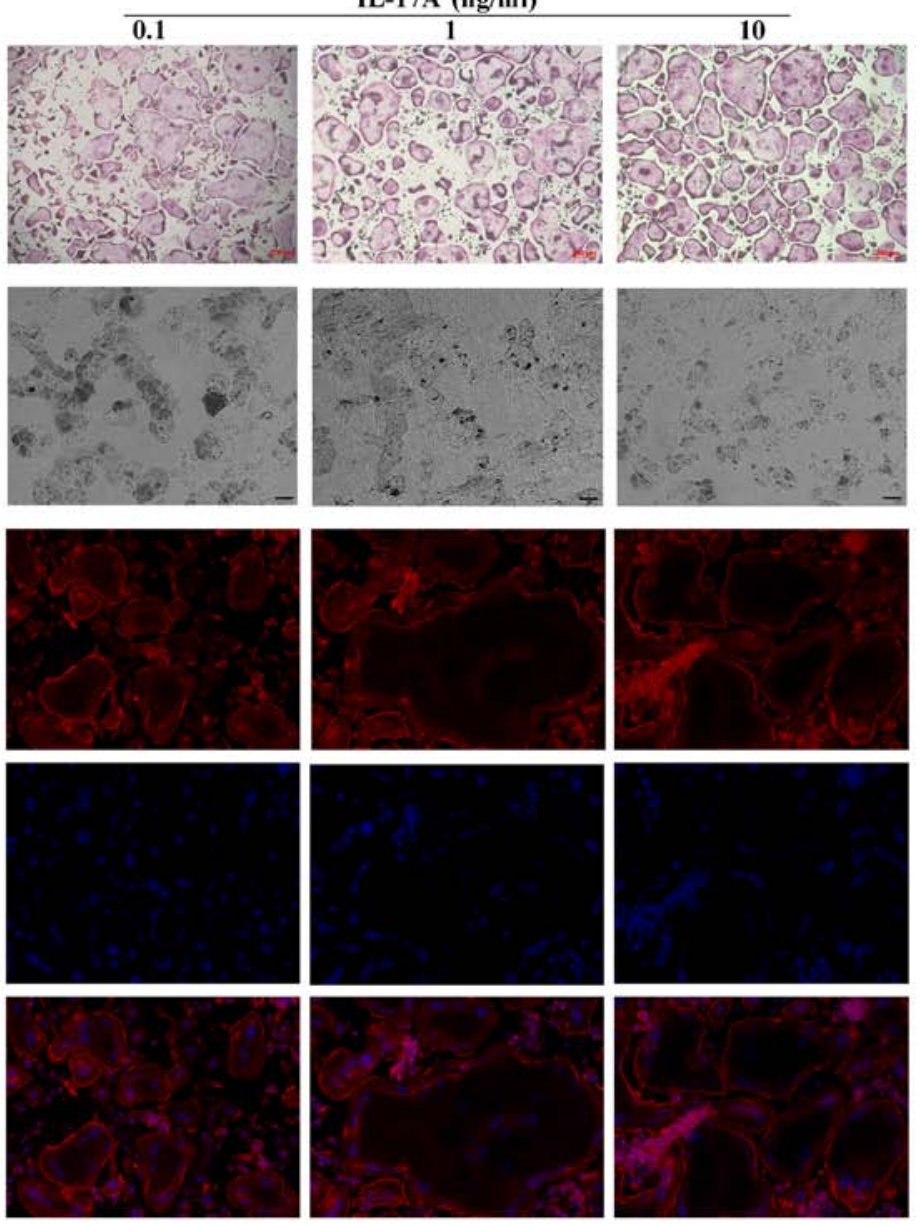

E

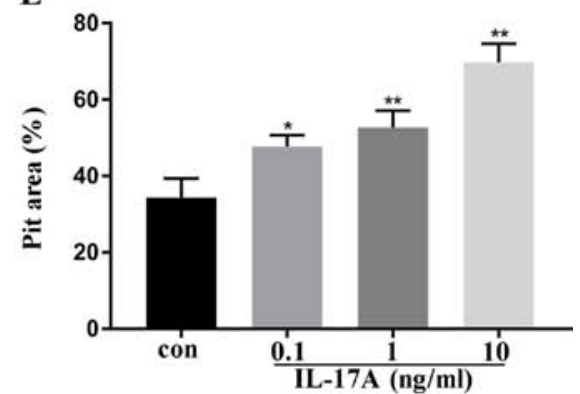

Figure 1. IL-17A exerts positive effects on osteoclast formation in bone marrow macrophages in vitro. (A) The representative TRAP ${ }^{+}$multinucleated cells/well are presented (magnification, x10), scale bar, $200 \mu \mathrm{m}$. (B) The resorption pits of the bone slices were visualized with a scanning electron microscope (magnification, x200) scale bar, $50 \mu \mathrm{m}$. (C) Immunofluorescence staining was performed with phalloidin (red) and DAPI (blue). Images were obtained and analyzed using a Leica fluorescence microscope (magnification, $\mathrm{x} 40$ ) scale bar, $20 \mu \mathrm{m}$. (D) Quantitative analysis was implemented to assess the TRAP ${ }^{+}$multinucleated cells/well. (E) Quantitative analysis was performed on resorption pit areas. ${ }^{*} \mathrm{P}<0.05,{ }^{* * *} \mathrm{P}<0.01,{ }^{* * *} \mathrm{P}<0.001$ compared to the control group (mean \pm standard deviation). IL-17A, interleukin IL-17A; TRAP, tartrate-resistant acid phosphatase; F-actin, filamentous actin; con, control group.

Osteoclasts are exclusive bone resorbing cells that could secrete lysosomal enzymes into the extracellular space to degrade bone matrix (24). The effects of IL-17A, a pro-inflammatory cytokine, were examined on osteoclast differentiation and bone resorption in vitro. It was found that the number of $\mathrm{TRAP}^{+}$multinucleated cells, and the formation of actin rings accompanied by the pit area of bone resorption were increased in response to increased concentrations of IL-17A, thereby indicating that IL-17A could facilitate osteoclast differentiation and bone resorption. A similar result was deduced that IL-17 exhibited a positive effect on osteoclast differentiation of human-derived bone marrow cells (29). Further study revealed that IL-17 upregulated the expression of RANK on human osteoclast precursors to enhance the sensitivity to RANKL signaling, culminating in bone resorption (30). Notably, the differentiation of osteoclasts from macrophage precursors is dependent on the presence of RANKL, and RANKL-RANK interaction is essential for the activation of various signaling cascades of osteoclastogenesis $(26,31)$. Considering these findings, it was speculated that in the present study, the strong enhancement of osteoclast activity promoted by IL-17A of BMMs is attributed to the synergistic effect with RANKL. 

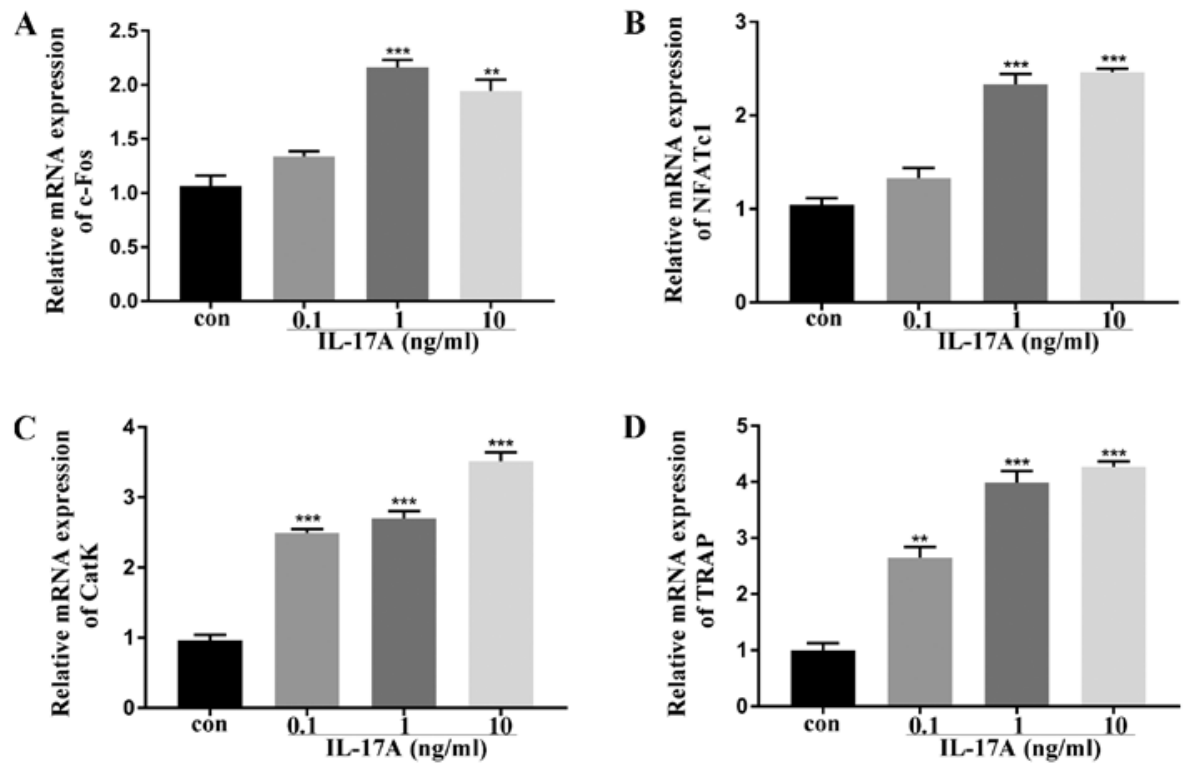

Figure 2. Effects of IL-17A on the expression of osteoclast-related genes involved in osteoclastogenesis. The mRNA expression of (A) $c$-Fos and (B) NFATc1 was detected on day 1 with different concentrations of IL-17A. The mRNA expression of (C) CatK and (D) TRAP was detected after 3 days of different concentrations of IL-17A. Data were normalized to GAPDH expression and presented as a fold-change relative to the control group (mean \pm standard deviation). ${ }^{* * *} \mathrm{P}<0.01$, ${ }^{* * * *} \mathrm{P}<0.001$ compared to the control group. IL-17A, interleukin IL-17A; NFAcT1, nuclear factor of activated T-cells, cytoplasmic 1; TRAP, tartrate-resistant acid phosphatase; CatK, cathepsin K; GAPDH, glyceraldehyde-3-phosphate dehydrogenase; con, control group.

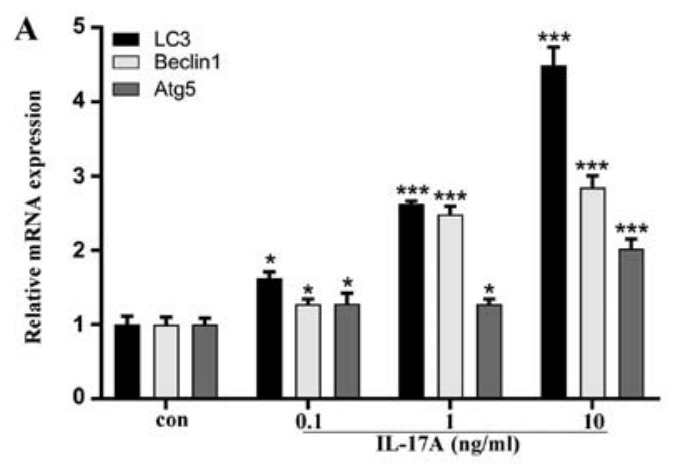

B

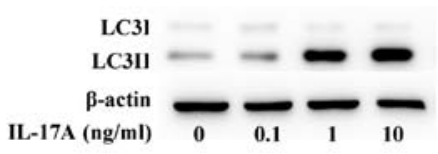

D c

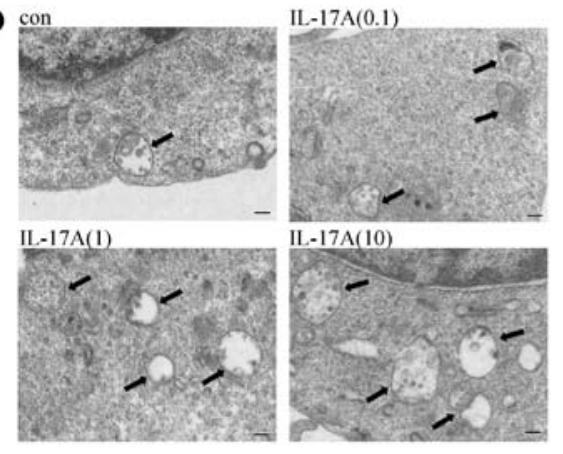

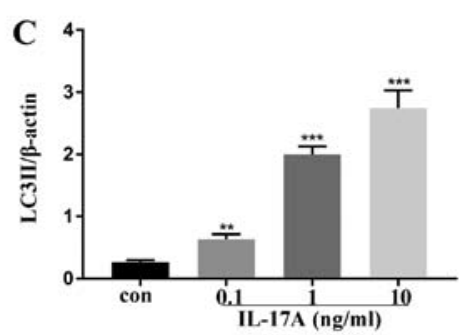

E

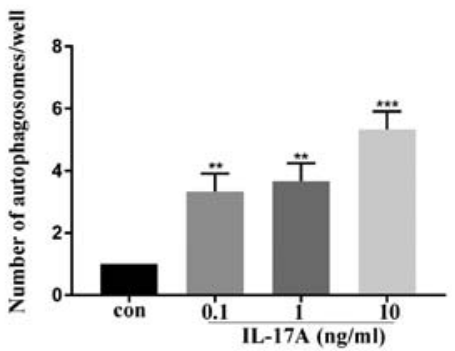

Figure 3. IL-17A stimulates osteoclast formation by inducing autophagy. (A) The mRNA expression of autophagy-related genes was determined using real-time quantitative polymerase chain reaction with IL-17A treatment after $48 \mathrm{~h}$ (mean \pm standard deviation). (B) The expression of LC3 protein during IL-17A-mediated osteoclastogenesis was detected using western blot analysis. (C) Densitometric analysis of LC3-II expression. (D) Assessment of autophagy activity by transmission electron microscopy. Typical observations depict autophagosomes. (E) The quantitation of the number of autophagosomes (mean \pm standard deviation). ${ }^{*} \mathrm{P}<0.05,{ }^{* *} \mathrm{P}<0.01,{ }^{* * * *} \mathrm{P}<0.001$ compared to the control group. IL-17A, interleukin IL-17A; LC3, microtubule-associated protein 1 light chain 3; con, control group. 
A

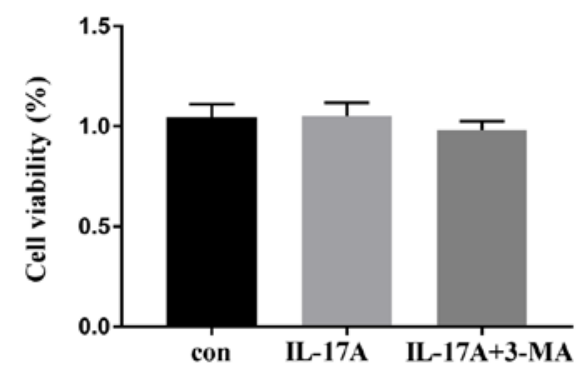

C

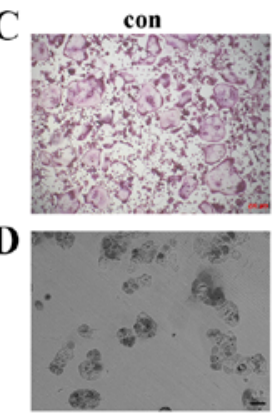

F

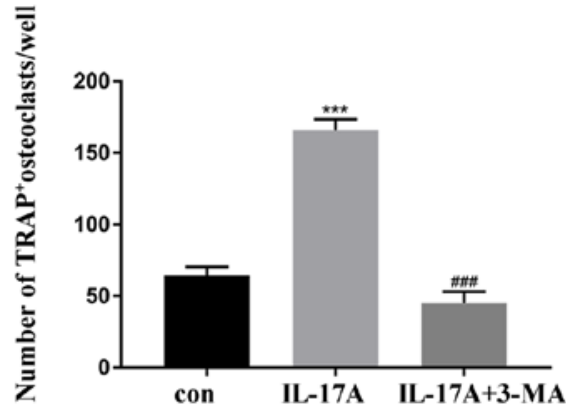

H
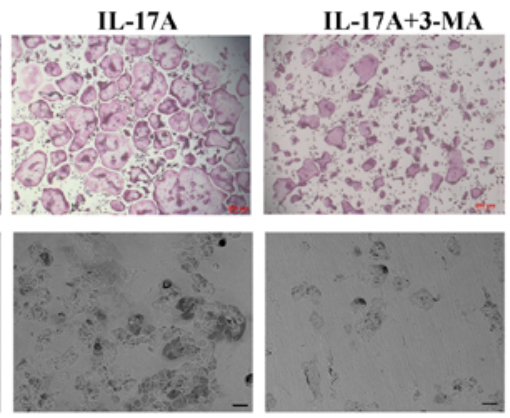

con

B

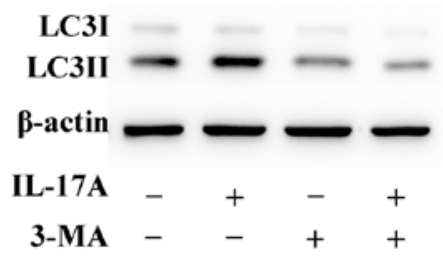

E

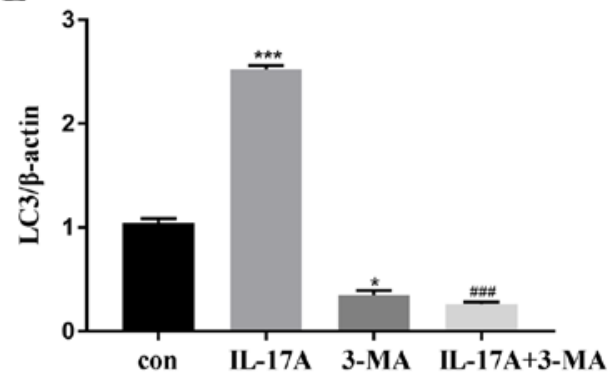

G
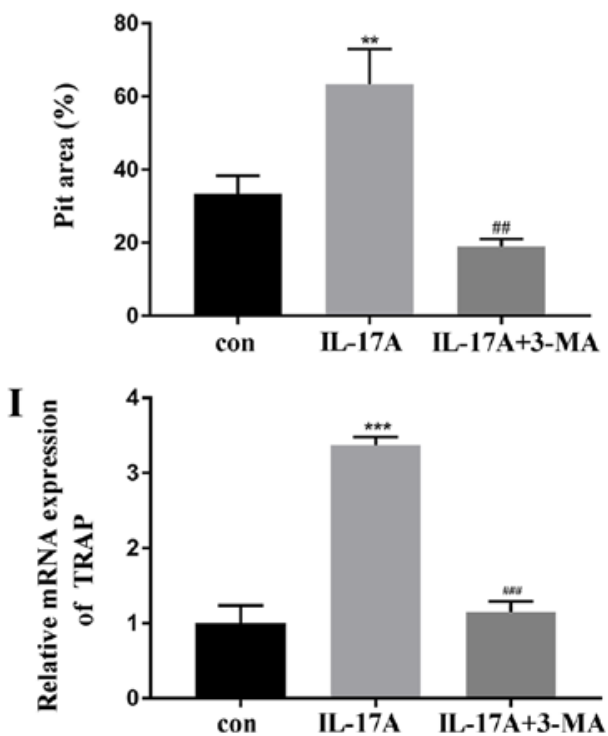

Figure 4. Inhibition of autophagy abrogates IL-17A-mediated osteoclastogenesis. (A) Cell viability was measured by CCK-8 assay. BMMs were incubated with or without IL-17A $(10 \mathrm{ng} / \mu \mathrm{l})$ for $48 \mathrm{~h}$, and 3-MA $(10 \mathrm{nM})$ was added into IL-17A medium. (B) Western blot analysis was used to analyze the expression of LC3. (C) Fixed cells were stained for TRAP, and representative TRAP ${ }^{+}$multinucleated cells/well are displayed. Magnification, x10; Scale bar, $200 \mu \mathrm{m}$. (D) The resorption pits of the bone slices were visualized using a scanning electron microscope after 10 days of culture. Magnification, x200; scale bar, $50 \mu \mathrm{m}$. (E) Densitometric analysis of LC3-II expression. The cells were pretreated with 3-MA (2 nM) for $2 \mathrm{~h}$, and then, with RANKL (50 ng/ $\mu$ l) plus IL-17A $(10 \mathrm{ng} / \mu \mathrm{l})$ for 5 days. (F) Quantitative analysis was implemented to assess the TRAP ${ }^{+}$multinucleated cells/well. (G) Quantitative analysis was performed on resorption pit areas. The osteoclast-related genes (H) CatK and (I) TRAP were analyzed by real-time quantitative polymerase chain reaction. Data were normalized to the GAPDH expression and presented as the fold-change relative to the control group (mean \pm standard deviation). ${ }^{*} \mathrm{P}<0.05,{ }^{* *} \mathrm{P}<0.01,{ }^{* * * *} \mathrm{P}<0.001$ compared to the control group. ${ }^{\# \#} \mathrm{P}<0.01,{ }^{\# \# \#} \mathrm{P}<0.001$, compared to the IL-17A-treated group. IL-17A, interleukin IL-17A; 3-MA, 3-methyladenine; LC3, microtubule-associated protein 1 light chain 3; CatK, cathepsin K; TRAP, tartrate-resistant acid phosphatase; RANKL, receptor activator of nuclear factor- $\mathrm{\kappa B}$ ligand; GAPDH, glyceraldehyde-3-phosphate dehydrogenase; con, control group.

However, Kitami et al (32) observed suppression of osteoclast differentiation of RAW264.7 as a result of the treatment of IL-17. IL-17 markedly suppressed the osteoclastogenesis of canine bone marrow-derived macrophages at a maximal concentration (33). Collectively, these studies contradicted the present experimental results, which may be attributed to various factors, including different experimental conditions and species. Therefore, theories on the activity of IL-17A in osteoclast differentiation are yet controversial, and thus, exploring the specific underlying mechanisms is imperative.
Osteoclast-specific genes contribute to various stages of osteoclast formation and activation (26). c-Fos is one of the key transcription factors in the AP-1 family, which is required for the early phase of osteoclast differentiation (34). NFATc1 is the hallmark of osteoclast fate, and is essential for the survival and differentiation of osteoclast precursors (35). CatK and TRAP are largely regarded as terminal osteoclast differentiation markers and highly expressed by activated osteoclasts to mediate the function of bone resorption $(36,37)$. Moreover, mature osteoclasts exhibit bone resorption capacity 


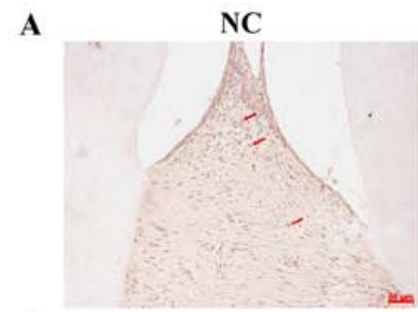

C

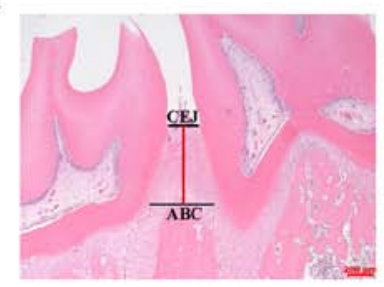

$\mathbf{E}$

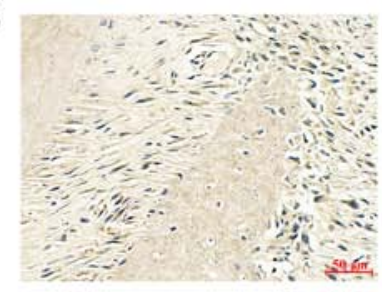

G

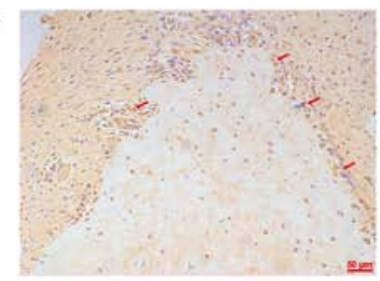

NS
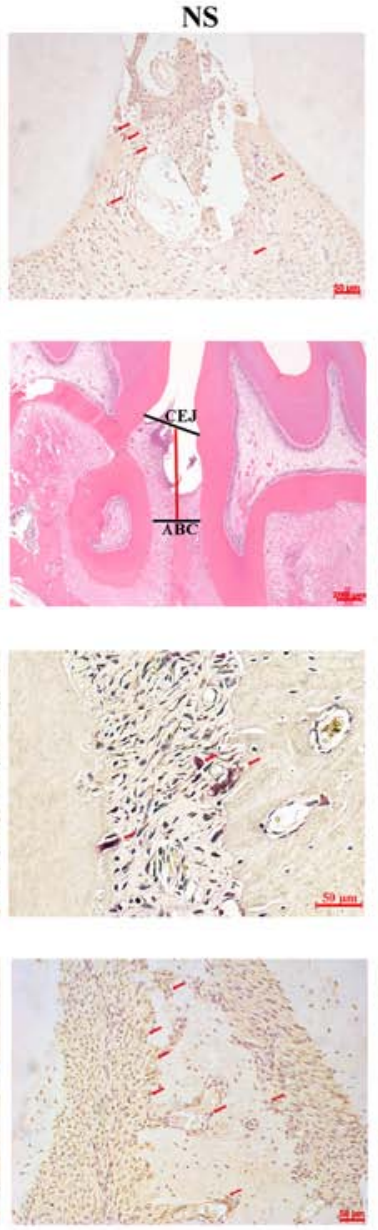

IL-17
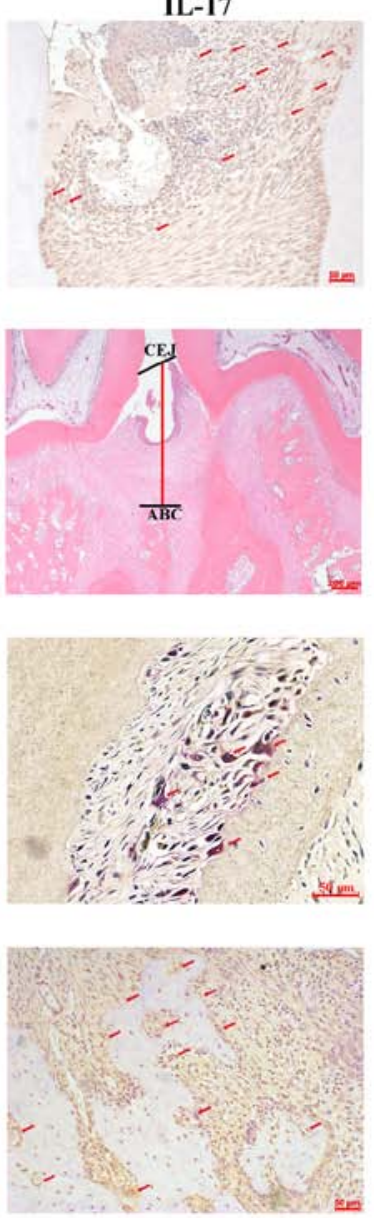

B

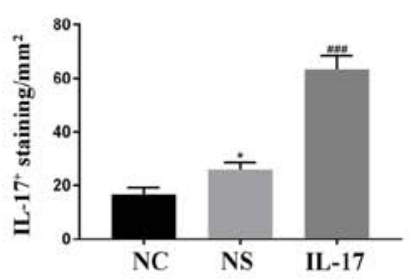

D

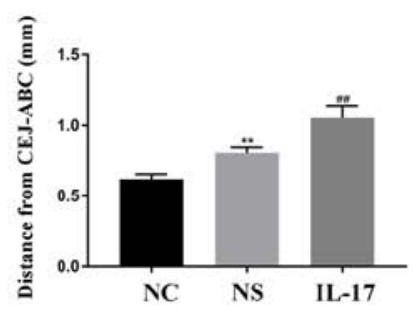

$\mathbf{F}$

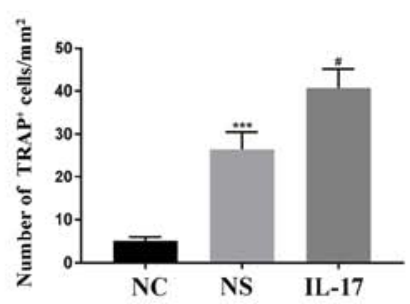

H

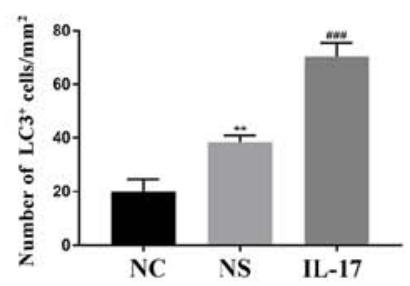

Figure 5. Effects of IL-17A on osteoclast activity in vivo. (A) Immunohistochemical staining was used to determine the level of IL-17A expression (arrows). Scale bar, $50 \mu \mathrm{m}$. (B) IL-17A expression was analyzed quantitatively. (C) The distance between CEJ and ABC (red line) was observed by H\&E staining. Scale bar, $200 \mu \mathrm{m}$. (D) Quantitative analysis was performed on the distance. (E) TRAP staining was used to detect the osteoclasts (red arrows). Scale bar, $50 \mu \mathrm{m}$. (F) Quantitative analysis was performed on the number of TRAP ${ }^{+}$cells. (G) Immunohistochemical staining was used to examine the level of LC3 expression (arrows). Scale bar, $50 \mu \mathrm{m}$. (H) Quantitative analysis was performed on LC3 level. ${ }^{*} \mathrm{P}<0.05,{ }^{* *} \mathrm{P}<0.01,{ }^{* * *} \mathrm{P}<0.001$ compared to the $\mathrm{NC}$ group. ${ }^{\#} \mathrm{P}<0.05,{ }^{\# \#} \mathrm{P}<0.01$,

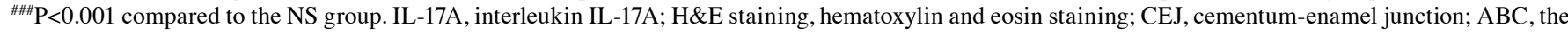
alveolar callus; TRAP, tartrate-resistant acid phosphatase; LC3, microtubule-associated protein 1 light chain 3; NC, normal control group; NS, normal saline group; IL-17A, IL-17A group.

by secreting protons, CatK and so forth. In the present study, the expression of $c$-Fos, NFATcl, CatK, and TRAP genes was increased with the treatment of IL-17A, indicating that IL-17A could promote the osteoclast differentiation in BMMs.

Autophagy, a conserved lysosomal degradation process, is essential for cell survival, differentiation, and development and is regulated by some autophagy-related genes (38). Emerging evidence has revealed that autophagy was activated in response to several pro-inflammatory cytokines, such as IL-1 $\beta$ and TNF $\alpha$ accompanied by a robust increase in osteoclast differentiation and bone resorption $(18,39)$. The present findings supported the hypothesis that IL-17A, a pro-inflammatory cytokine, enhanced the autophagic activity including the increased expression of an autophagy-related gene, accumulation of LC3-II, as well as autophagosome formation along with osteoclast differentiation. Moreover, the addition of autophagy inhibitor 3-MA could reduce the level of osteoclast-related markers, thereby attenuating the osteoclast formation and bone resorption after the treatment with IL-17A. Thus, this result confirmed our speculation that autophagy exhibits the positive effect on osteoclast activity under IL-17 stimulation.

In addition, Ke et al (40) observed that IL-17 facilitated osteoclast precursor autophagy at a low concentration, and the activation of the RANKL/JNK pathway may be involved in IL-17-enhanced osteoclastogenesis. In the present study, it was also revealed that p-JNK and p-p38 were increased after addition of IL-17 (data not shown), which suggested that mitogen-activated protein kinase (MAPK) pathways including JNK and p38 may mediate autophagic activity of osteoclasts after addition of IL-17. However, more specific mechanisms require revealing to elucidate which pathway may exist in IL-17-enhanced autophagy and osteoclastogenesis.

It is well accepted that autophagy, as a highly conserved intracellular metabolic mechanism, has an anti-apoptotic effect. Xue et al (41), revealed that IL-17 modulated apoptosis of osteoclast precursors, RAW264.7, to influence osteoclastogenesis. However, RAW264.7 is a cell line which differs from the primary mouse bone marrow macrophages that we used (42). Therefore, whether IL-17A has an effect on the apoptosis of 
osteoclasts from primary bone marrow macrophages requires further study.

Notably, recent studies have demonstrated that the knockdown of Beclin1 (43) or p62 (44) using small interfering RNA (siRNA) resulted in the inhibition of osteoclastogenesis, and further deletion of Atg7 prevented the TNF $\alpha$-induced bone loss in mice (45). Similar results revealed that the pharmacological or genetic inactivation of autophagy could ameliorate bone loss by inhibiting osteoclast differentiation. This phenomenon could implicate the direct translation targeting autophagy, which in turn, would provide promising therapeutic approaches in the prevention and treatment of inflammatory bone resorption.

Nonetheless, the number of osteoclasts was increased along with the exacerbation of alveolar bone resorption in response to IL-17A treatment in a rat periodontitis model. A previous study revealed that $I L-17 A$ gene transfer increased the level of osteoclasts and biomarkers of bone resorption in collagen-induced arthritis, indicating that IL-17A is crucial for pathological bone loss via direct activation of osteoclasts (46). Further studies demonstrated that blocking of IL-17 with IL-17 antibody or IL-17-deficient mice suppressed joint damage in experimental arthritis, rendering IL-17 as an intervention target for inflammatory bone resorption in rheumatoid arthritis (12,47-49). Notably, rheumatoid arthritis exhibits specific similarities with periodontitis with respect to inflammatory diseases, wherein inflammatory cytokines accelerate bone and joint destruction (50). However, although IL-17 is ascribed to joint disruption, the specific mechanism in periodontitis has not been reported yet, necessitating further investigation.

In conclusion, the present study revealed that IL-17A directly promoted osteoclast differentiation and exacerbated bone resorption in vitro and in vivo, and autophagic activity was involved in the IL-17A-mediated osteoclast differentiation of BMMs. These findings contribute to the molecular mechanism underlying IL-17A in alveolar bone destruction, and provide insight into the clinical therapeutic targets for periodontitis. However, these rudimentary studies cannot completely elucidate the complex correlation between the immune system and alveolar bone destruction. Future studies should be primarily focused on the specific mechanism of IL-17A in periodontitis in order to investigate the systemic treatment by blocking IL-17 for the prevention of periodontitis.

\section{Acknowledgements}

Not applicable.

\section{Funding}

The present study was supported by the National Natural Science Foundation of China (grant nos. 81771072 and 81800972), and the Natural Science Foundation of Zhejiang Province (grant nos. LY18H140002 and LY15H140003).

\section{Availability of data and materials}

The datasets used and analyzed during the present study are available from the corresponding author on reasonable request.

\section{Authors' contributions}

LS and JT performed the experiments, analyzed the data and wrote the manuscript. ZW cultured the cells and analyzed the data. MX performed western blotting and analyzed data. QT and YW analyzed data and revised the manuscript. LC conceived and designed the study. PD designed the study and revised the manuscript. All authors read and approved the manuscript and agree to be accountable for all aspects of the research in ensuring that the accuracy or integrity of any part of the work are appropriately investigated and resolved.

\section{Ethics approval and consent to participate}

All mice and rat experiments were performed in accordance with the principles and procedures of the National Institutes of Health (NIH) for the Care and Use of Laboratory Animals. The approval number granted by the Animal Ethics Committee of The Second Affiliated Hospital of Zhejiang University School of Medicine is 2017-052. All surgeries were performed under sodium pentobarbital anesthesia, and all efforts were made to minimize suffering.

\section{Patient consent for publication}

Not applicable.

\section{Competing interests}

The authors declare that they have no competing interests.

\section{References}

1. Page RC and Kornman KS: The pathogenesis of human periodontitis: An introduction. Periodontol 2000 14: 9-11, 1997.

2. Hajishengallis G: Immunomicrobial pathogenesis of periodontitis: Keystones, pathobionts, and host response. Trends Immunol 35: 3-11, 2014

3. Cochran DL: Inflammation and bone loss in periodontal disease. J Periodontol 79 (8 Suppl): S1569-S1576, 2008.

4. Cheng WC, Hughes FJ and Taams LS: The presence, function and regulation of IL-17 and Th17 cells in periodontitis. J Clin Periodontol 41: 541-549, 2014.

5. Chen XT, Chen LL, Tan JY, Shi DH, Ke T and Lei LH: Th17 and Th1 lymphocytes are correlated with chronic periodontitis. Immunol Invest 45: 243-254, 2016

6. Chen XT, Tan JY, Lei LH and Chen LL: Cytokine levels in plasma and gingival crevicular fluid in chronic periodontitis. Am J Dent 28: 9-12, 2015.

7. Cardoso CR, Garlet GP, Crippa GE, Rosa AL, Junior WM, Rossi MA and Silva JS: Evidence of the presence of T helper type 17 cells in chronic lesions of human periodontal disease. Oral Microbiol Immunol 24: 1-6, 2009.

8. da Costa TA, Silva MJ, Alves PM, Chica JE, Barcelos EZ, Giani MA, Garlet GP, Silva JS, Junior VR, Rodrigues DB and Cardoso CR: Inflammation biomarkers of advanced disease in nongingival tissues of chronic periodontitis patients. Mediators Inflamm 2015: 983782, 2015.

9. Hienz SA, Paliwal S and Ivanovski S: Mechanisms of bone resorption in periodontitis. J Immunol Res 2015: 615486, 2015.

10. Fossiez F, Djossou O, Chomarat P, Flores-Romo L, Ait-Yahia S, Maat C, Pin JJ, Garrone P, Garcia E, Saeland S, et al: T cell interleukin-17 induces stromal cells to produce proinflammatory and hematopoietic cytokines. J Exp Med 183: 2593-2603, 1996.

11. Jovanovic DV, Di Battista JA, Martel-Pelletier J, Jolicoeur FC, He Y, Zhang M, Mineau F and Pelletier JP: IL-17 stimulates the production and expression of proinflammatory cytokines, IL-beta and TNF-alpha, by human macrophages. J Immunol 160: 3513-3521, 1998. 
12. Lubberts E, van den Bersselaar L, Oppers-Walgreen B, Schwarzenberger P, Coenen-de Roo CJ, Kolls JK, Joosten LA and van den Berg WB: IL-17 promotes bone erosion in murine collagen-induced arthritis through loss of the receptor activator of NF-kappa B ligand/osteoprotegerin balance. J Immunol 170: 2655-2662, 2003.

13. Feng Y, He D, Yao Z and Klionsky DJ: The machinery of macroautophagy. Cell Res 24: 24-41, 2014.

14. Hale AN, Ledbetter DJ, Gawriluk TR and Rucker EB III Autophagy: Regulation and role in development. Autophagy 9: 951-972, 2013

15. Pierrefite-Carle V, Santucci-Darmanin S, Breuil V, Camuzard O and Carle GF: Autophagy in bone: Self-eating to stay in balance. Ageing Res Rev 24: 206-217, 2015.

16. Gelman A and Elazar Z: Autophagic factors cut to the bone. Dev Cell 21: 808-810, 2011.

17. DeSelm CJ, Miller BC, Zou W, Beatty WL, van Meel E, Takahata Y, Klumperman J, Tooze SA, Teitelbaum SL and Virgin HW: Autophagy proteins regulate the secretory component of osteoclastic bone resorption. Dev Cell 21: 966-974, 2011

18. Chung YH, Choi B, Song DH, Song Y, Kang SW, Yoon SY, Kim SW, Lee HK and Chang EJ: Interleukin-1 $\beta$ promotes the LC3-mediated secretory function of osteoclast precursors by stimulating the $\mathrm{Ca} 2+-$ dependent activation of ERK. Int J Biochem Cell Biol 54: 198-207, 2014.

19. Lin NY, Stefanica A and Distler JH: Autophagy: A key pathway of TNF-induced inflammatory bone loss. Autophagy 9: 1253-1255 2013.

20. Xing L and Boyce BF: RANKL-based osteoclastogenic assays from murine bone marrow cells. Methods Mol Biol 1130 307-313, 2014.

21. Livak KJ and Schmittgen TD: Analysis of relative gene expression data using real-time quantitative PCR and the 2(-Delta Delta C(T)) method. Methods 25: 402-408, 2001.

22. Zhang Y, Xiong Y, Chen X, Chen C, Zhu Z and Li L: Therapeutic effect of bone marrow mesenchymal stem cells pretreated with acetylsalicylic acid on experimental periodontitis in rats. Int Immunopharmacol 54: 320-328, 2018

23. Castro ML, Franco GC, Branco-de-Almeida LS, Anbinder AL, Cogo-Muller K, Cortelli SC, Duarte S, Saxena D and Rosalen PL: Downregulation of proteinase-activated receptor-2, interleukin-17, and other proinflammatory genes by subantimicrobial doxycycline dose in a rat periodontitis model. J Periodontol 87: 203-210, 2016

24. Boyle WJ, Simonet WS and Lacey DL: Osteoclast differentiation and activation. Nature 423: 337-342, 2003.

25. Teitelbaum SL and Ross FP: Genetic regulation of osteoclast development and function. Nat Rev Genet 4: 638-649, 2003.

26. Asagiri $\mathrm{M}$ and Takayanagi $\mathrm{H}$ : The molecular understanding of osteoclast differentiation. Bone 40: 251-264, 2007.

27. Cekici A, Kantarci A, Hasturk H and Van Dyke TE: Inflammatory and immune pathways in the pathogenesis of periodontal disease. Periodontol 2000 64: 57-80, 2014

28. Tompkins KA: The osteoimmunology of alveolar bone loss Connect Tissue Res 57: 69-90, 2016.

29. Sprangers S, Schoenmaker T, Cao Y, Everts V and de Vries TJ: Different blood-borne human osteoclast precursors respond in distinct ways to IL-17A. J Cell Physiol 231: 1249-1260, 2016.

30. Adamopoulos IE, Chao CC, Geissler R, Laface D, Blumenschein W, lwakura Y, McClanahan T and Bowman EP. Interleukin-17A upregulates receptor activator of NF-kappaB on osteoclast precursors. Arthritis Res Ther 12: R29, 2010.

31. Boyce BF: Advances in the regulation of osteoclasts and osteoclast functions. J Dent Res 92: 860-867, 2013.

32. Kitami S, Tanaka H, Kawato T, Tanabe N, Katono-Tani T, Zhang F, Suzuki N, Yonehara Y and Maeno M: IL-17A suppresses the expression of bone resorption-related proteinases and osteoclast differentiation via IL-17RA or IL-17RC receptors in RAW264.7 cells. Biochimie 92: 398-404, 2010.

33. Wijekoon S, Bwalya EC, Fang J, Kim S, Hosoya K and Okumura M: Chronological differential effects of pro-inflammatory cytokines on RANKL-induced osteoclast differentiation of canine bone marrow-derived macrophages. J Vet Med Sci 79: 2030-2035, 2017.
34. Grigoriadis AE, Wang ZQ, Cecchini MG, Hofestetter W, Felix R, Fleisch HA and Wanger EF: c-Fos: A key regulator of osteoclast-macrophage lineage determination and bone remodeling. Science 266: 443-448, 1994

35. Asagiri M, Sato K, Usami T, Ochi S, Nishina H, Yoshida H, Morita I, Wagner EF, Mark TW, Serfling E and Takayanagi H: Autoamplification of NFATc1 expression determines its essential role in bone homeostasis. J Exp Med 202: 1261-1269, 2005.

36. Costa AG, Cusano NE, Silva BC, Cremers S and Bilezikian JP: Cathepsin K: Its skeletal actions and role as a therapeutic target in osteoporosis. Nat Rev Rheumatol 7: 447-456, 2011.

37. Hayman AR: Tartrate-resistant acid phosphatase (TRAP) and the osteoclast/immune cell dichotomy. Autoimmunity 41: 218-223, 2008.

38. Yang Z and Klionsky DJ: Eaten alive: A history of macroautophagy. Nat Cell Biol 12: 814-822, 2010.

39. Lin NY, Beyer C, Giessl A, Kireva T, Scholtysek C, Uderhardt S, Munoz LE, Dees C, Distler A, Wirtz S, et al: Autophagy regulates $\mathrm{TNF} \alpha$-mediated joint destruction in experimental arthritis. Ann Rheum Dis 72: 761-768, 2013.

40. Ke D, Fu X, Xue Y, Wu H, Zhang Y, Chen X and Hou J: IL-17A regulates the autophagic activity of osteoclast precursors through RANKL-JNK1 signaling during osteoclastogenesis in vitro. Biochem Biophys Res Commun 497: 890-896, 2018.

41. Xue Y, Liang Z, Fu X, Wang T, Xie Q and Ke D: IL-17A modulates osteoclast precursors' apoptosis through autophagy-TRAF3 signaling during osteoclastogenesis. Biochem Biophys Res Commun 508: 1088-1092, 2019.

42. Ng AY, Tu C, Shen S, Xu D, Oursler MJ, Qu J and Yang S: Comparative characterization of osteoclasts derived from murine bone marrow macrophages and RAW 264.7 cells using quantitative proteomics. JBMR Plus 2: 328-340, 2018.

43. Chung YH, Jang Y, Choi B, Song DH, Lee EJ, Kim SM, Song Y, Kang SW, Yoon SY and Chang EJ: Beclin-1 is required for RANKL-induced osteoclast differentiation. J Cell Physiol 229: 1963-1971, 2014.

44. Li RF, Chen G, Ren JG, Zhang W, Wu ZX, Liu B, Zhao Y and Zhao YF: The adaptor protein p62 is involved in RANKL-induced autophagy and osteoclastogenesis. J Histochem Cytochem 62: 879-888, 2014

45. Lin NY, Chen CW, Kagwiria R, Liang R, Beyer C, Distler A, Luther J, Engelke K, Schett G and Distler JH: Inactivation of autophagy ameliorates glucocorticoid-induced and ovariectomy-induced bone loss. Ann Rheum Dis 75: 1203-1210, 2016.

46. Adamopoulos IE, Suzuki E, Chao CC, Gorman D, Adda S, Maverakis E, Zarbalis K, Geissler R, Asio A, Blumenschein WM, et al: IL-17A gene transfer induces bone loss and epidermal hyperplasia associated with psoriatic arthritis. Ann Rheum Dis 74: 1284-1292, 2015.

47. Park MJ, Park HS, Oh HJ, Lim JY, Yoon BY, Kim HY, Cho ML and Cho SG: IL-17-deficient allogeneic bone marrow transplantation prevents the induction of collagen-induced arthritis in DBA/1J mice. Exp Mol Med 44: 694-705, 2012.

48. Koenders MI, Lubberts E, Oppers-Walgreen B, van den Bersselaar L, Helsen MM, Di Padova FE, Boots AM, Gram H, Joosten LA and van den Berg WB: Blocking of interleukin-17 during reactivation of experimental arthritis prevents joint inflammation and bone erosion by decreasing RANKL and interleukin-1. Am J Pathol 167: 141-149, 2005

49. Nakae S, Nambu A, Sudo K and Iwakura Y: Suppression of immune induction of collagen-induced arthritis in IL-17-deficient mice. J Immunol 171: 6173-6177, 2003.

50. Araujo VM, Melo IM and Lima V: Relationship between periodontitis and rheumatoid arthritis: Review of the Literature. Mediators Inflamm 2015: 259074, 2015.

This work is licensed under a Creative Commons Attribution-NonCommercial-NoDerivatives 4.0 International (CC BY-NC-ND 4.0) License. 